\title{
Predicting Drusen Regression from OCT in Patients with Age-Related Macular Degeneration
}

\author{
Hrvoje Bogunović, Alessio Montuoro, Sebastian M. Waldstein, \\ Magdalena Baratsits, Ferdinand Schlanitz, and Ursula Schmidt-Erfurth \\ Christian Doppler Laboratory for Ophthalmic Image Analysis, Vienna Reading \\ Center, Department of Ophthalmology, Medical University of Vienna, Austria
}

\begin{abstract}
Age-related macular degeneration (AMD) is a leading cause of blindness in developed countries. The presence of drusen is the hallmark of early/intermediate AMD, and their sudden regression is strongly associated with the onset of late AMD. In this work we propose a predictive model of drusen regression using optical coherence tomography (OCT) based features. First, a series of automated image analysis steps are applied to segment and characterize individual drusen and their development. Second, from a set of quantitative features, a random forest classifier is employed to predict the occurrence of individual drusen regression within the following 12 months. The predictive model is trained and evaluated on a longitudinal OCT dataset of 44 eyes from 26 patients using leave-one-patient-out cross-validation. The model achieved an area under the ROC curve of 0.81 , with a sensitivity of 0.74 and a specificity of 0.73 . The presence of hyperreflective foci and mean drusen signal intensity were found to be the two most important features for the prediction. This preliminary study shows that predicting drusen regression is feasible and is a promising step toward identification of imaging biomarkers of incoming regression.
\end{abstract}

\section{Introduction}

Age-related macular degeneration (AMD) is the leading cause of irreversible blindness in the elderly population in the developed world. Over time the disease relentlessly progresses toward late AMD, which appears in two forms: atrophic and neovascular AMD. The pathogenic mechanisms of AMD are still unclear and currently there is an effective treatment available only for the less common, neovascular form.

The introduction of optical coherence tomography (OCT) had a profound impact on the assessment, early detection, and monitoring of AMD progression by facilitating phenotyping of retina and its layers in fine detail, with micrometer resolution. Thus, to expedite the search for therapies which halt or reverse early/intermediate AMD it is essential to be able to identify pathomorphological changes and quantitatively evaluate AMD progression using adequate biomarkers accessible by OCT imaging.

A clinical hallmark and usually the first clinical finding of early AMD is the presence of drusen, deposits of cellular waste products that begin to accumulate

X. Chen, M. K. Garvin, J. Liu, E. Trucco, Y. Xu (Eds.): OMIA 2016, Held in Conjunction with MICCAI 2016, Athens, Greece, Iowa Research Online, pp. 41-48, 2016. Available from: http://ir.uiowa.edu/omia/2016_Proceedings/2016/ 


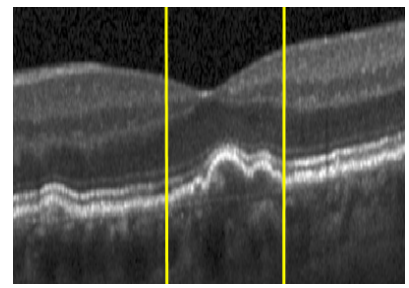

(a) $T_{0}$

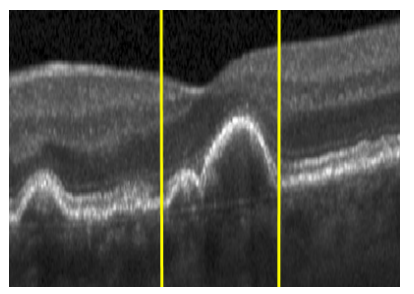

(b) $T_{\text {regression }}-3$ months

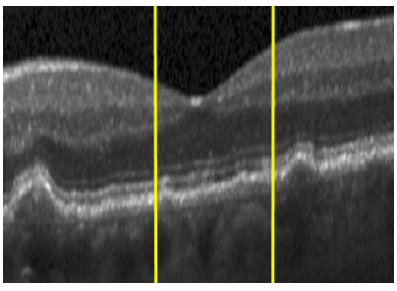

(c) $T_{\text {regression }}$

Fig. 1: Example of a drusen regression time course. (a) B-scan at baseline visit $T_{0}$, (b) last visit before the regression, and (c) first visit after the regression $T_{\text {regression }}$. Vertical lines (yellow) denote the same anatomical location.

between the retinal pigment epithelium (RPE) and the Bruch's membrane (BM). Drusen anatomic changes were found to correlate well with disease progression, hence drusen growth has often been suggested as a promising candidate for a surrogate biomarker of nonexudative AMD in clinical trials [1]. A drusen change of particular clinical interest is the drusen regression. It is a naturally occurring phenomenon, where drusen spontaneously dramatically decrease or completely disappear (Fig. 1). In many cases late AMD develops precisely at the location where the drusen regressed [2-4].

Early work on quantitative drusen analysis focused on drusen segmentation from OCT images as the first step. Most approaches focused on obtaining drusen by segmenting the surrounding surfaces denoting the RPE layer and the BM [58]. Those methods generally work well except for possible undersegmentation of very small or very large drusen. The subsequent steps consisted of quantifying and characterizing the drusen load in longitudinal studies for the purpose of estimating the risk of AMD progression $[2,9,10]$. Nevertheless, there is still a lack of sensitive and specific structural biomarkers predictive of late AMD at the individual patient level. Understanding the phenomenon of drusen regression evolved with studies observing natural history of AMD progression. In [2], the natural history is characterized with total drusen volume and area, but the regression could not be successfully predicted. In [3], the presence of hyperreflective foci (HRF) overlying drusen, and a heterogeneous internal reflectivity of these lesions were found to be related with the local atrophy onset in the ensuing months.

In this work, we propose a machine learning approach to predict whether individual druse will experience regression in the near future, i.e. the following 12 months. We utilize a novel OCT-based characterization of the outer retina using both the shape and the local appearance of its layered structure as well as their longitudinal development. The prediction method is evaluated using leaveone-patient-out cross-validation on a longitudinal dataset of OCT images of 44 eyes from 26 patients with early/intermediate AMD. 

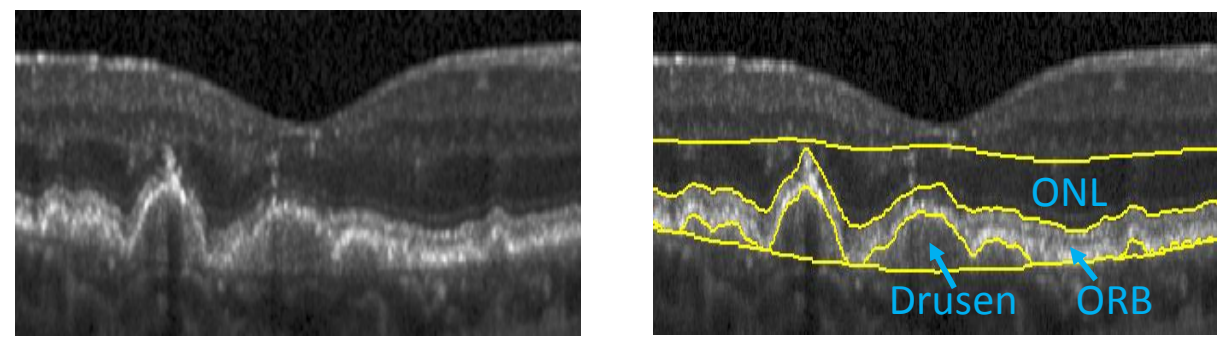

Fig. 2: Example of outer retina segmentation. Four surfaces are segmented (yellow) denoting three layers (blue).

\section{Methods}

The proposed methodology consists of the following processing pipeline. First, on OCT images we perform a layer segmentation of the outer retina with a specific focus on obtaining accurate drusen segmentation. Second, HRF are segmented by voxel classification of the outer retina. Subsequently, the longitudinal OCT scans of each eye are put into spatial correspondence by registering them to their baseline scan. Then, the footprints of individual drusen are identified from the baseline scan. Finally, features characterizing the shape, appearance at the baseline and their development at the follow-up visit are computed for each druse and are used to train a non-linear classifier to predict the occurrence of its regression within the following time-frame of 12 months.

\subsection{Outer Retinal Layer Segmentation}

The outer retinal layer segmentation (Fig. 2) is based on the Iowa Reference Algorithms [11,12], which we applied to obtain the segmentation of the outer nuclear layer (ONL). Then, we use the same graph-search segmentation approach with modified smoothness constraints, which define allowed change in surface height when moving between neighboring surface points. The lower RPE surface is obtained as a surface positioned on the bright to dark intensity gradient, below the ONL, with a weak smoothness constraint to allow for the deformations introduced by drusen. This defines the layer consisting of the outer retinal hyperreflective bands (ORB). Subsequently, to account for drusen, from the same cost function, the BM surface is obtained as a very smooth surface with strong smoothness constraints, analogous to the approach taken in [8].

\subsection{Hyperreflective Foci Segmentation}

To segment HRF (Fig. 3) a voxel classification based on unsupervised representation and auto-context is applied, analogous to the approach proposed in [13]. From a set of $2 \mathrm{D}$ image patches at various scales (ranging from $2 \times 2$ to $40 \times 40$ px) features are created using principal component analysis, where the first 15 

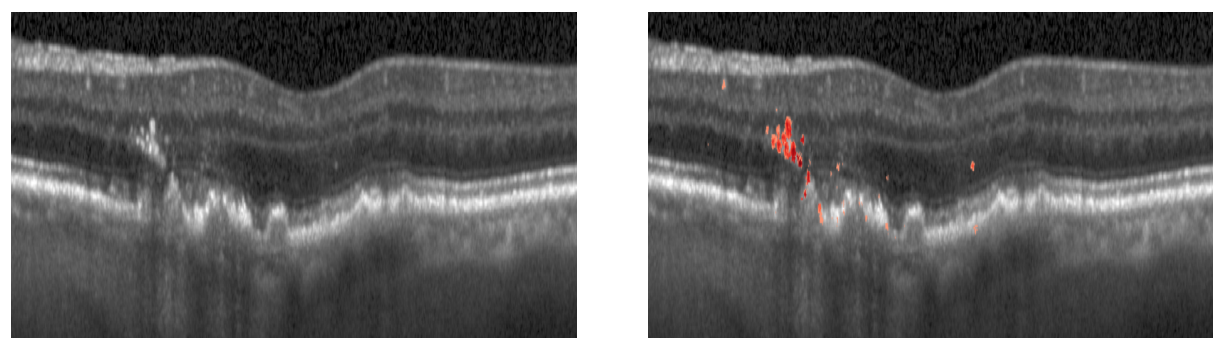

Fig. 3: Example of hyperreflective foci segmentation (in red).

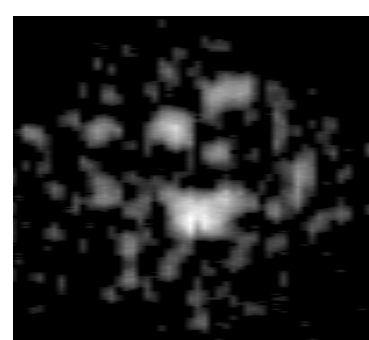

(a)

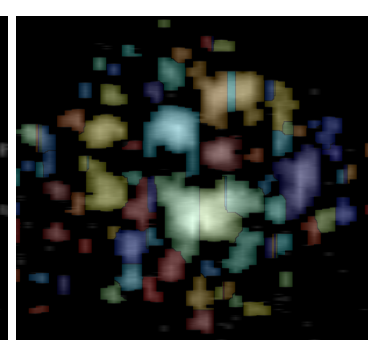

(b)

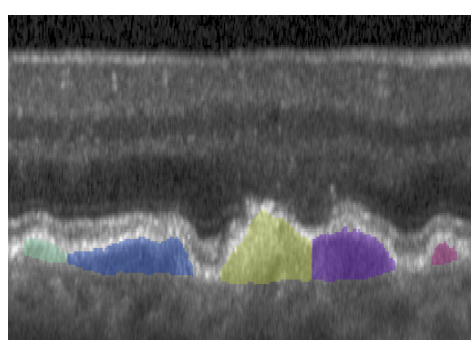

(c)

Fig. 4: Individual drusen segmentation. (a) Drusen thickness map. (b) Segmentation and labeling of individual drusen. (c) B-scan with confluent drusen segmented into individual drusen (e.g. yellow and purple).

eigenvectors are used as convolution kernels on the intensity scans. Then, from the convolutional features, a random forest classifier is trained to provide for each pixel of a B-scan the probability that it belongs to HRF. The results are further refined with auto-context, an iterative approach that includes spatial context extracted from previous classifications to refine the prediction result of the next iteration [14]. A set of $150 \mathrm{~B}$-scans from 40 OCT volumes where HRF was manually labeled was used as the training set, which was completely disjoint from the dataset used for the drusen regression prediction.

\subsection{Individual Drusen Segmentation}

From the segmentation of the outer retina (Subsection 2.1), thickness maps of the drusen layer are computed (Fig. 5). Local maximums of the thickness maps are taken to be the centers of individual drusen and are denoted as the foreground markers. All areas with the drusen thickness below an empirically defined threshold of $8 \mu \mathrm{m}$ are denoted as the background markers. To separate confluent drusen, the marker-controlled watershed segmentation is applied with the imposed foreground and background markers in the segmentation function. Example of the obtained individual drusen segmentation is shown on Fig. 4. 


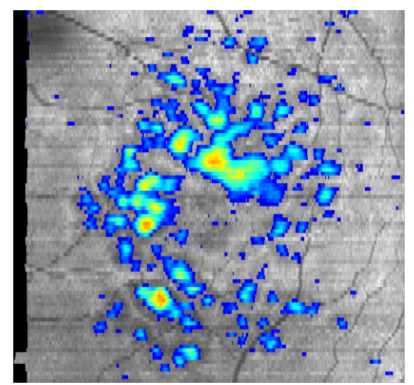

(a) $T_{0}$

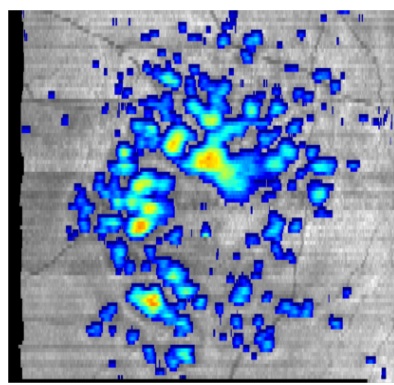

(b) $T_{0}+3$ months

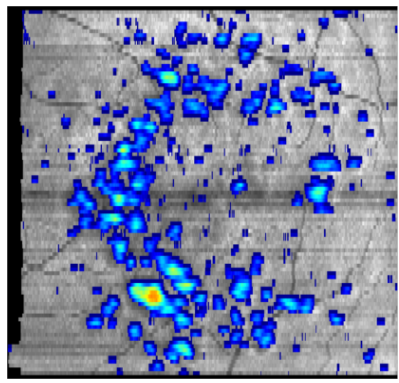

(c) $T_{0}+6$ months

Fig. 5: Aligned drusen thickness maps at (a) baseline visit $T_{0}$, (b) visit at month 3 , and (c) visit at month 6 when the drusen regression already occurred.

\subsection{Spatial Alignment}

To characterize drusen development across time, it is important to establish spatial alignment between the longitudinal scans of the same patient (Fig. 5). Before the OCT acquisition, confocal scanning laser ophthalmoscope (SLO) is used to acquire isotropic $2 \mathrm{D}$ fundus image of the same field of view, with superior spatial resolution than OCT. The SLO fundus image and the OCT image are acquired through the same optics and are co-registered by the device. To establish intra-patient spatial correspondence, we employ a keypoint-based registration between the SLO images. The keypoints are located using SURF feature detector, which is robust to substantial illumination changes. Finally, all scans of a patient are registered to its baseline scan using a similarity transform and the transformations resulting from the registration of SLO images are applied to the corresponding OCT images.

\subsection{Feature Extraction and Classification}

To capture drusen properties, we compute the following set of features from the baseline scan for each druse within the region of interest defined by its footprint:

Shape Druse height, area, volume and the mean thickness of the three segmented layers.

Intensity Internal reflectivity of the three segmented layers expressed as mean and std. dev. values of pixel intensities.

HRF Density and volume of HRF in ONL and ORB layers.

This results in 16 features per druse. In addition, differential features describing the development from baseline to the follow-up scan are computed, making a total of 32 features. The prediction model is built using random forest classifier, proved to be especially effective in classifying high dimensional and heterogeneous features. The forest has been grown with 200 trees and 4 features randomly sampled as candidates at each split of a tree. 


\section{Results}

The proposed method is evaluated on a longitudinal dataset consisting of 44 eyes from 26 patients with early and intermediate AMD enrolled in a prospective observational clinical study. The patients were monitored for a minimum of 1 year with 3 month follow-up intervals. The OCT imaging was performed with Spectralis SD-OCT (Heidelberg Engineering), which acquires anisotropic $3 \mathrm{D}$ images having $1024 \times 97 \times 496$ voxels with the size of $5.7 \times 60.5 \times 3.87 \mathrm{\mu m}^{3}$, covering the volume of $6 \times 6 \times 2 \mathrm{~mm}^{3}$. In addition, the device acquires SLO image with $1536 \times 1536$ pixels of size $5.7 \times 5.7 \mathrm{\mu m}^{2}$. Even though Spectralis device has a rescan feature, allowing follow-up scans to be acquired at the same location as the prior scans, it was not always utilized hence the need for the spatial alignment by the developed image registration method.

Given the large number of segmented drusen at the baseline (589 in total), the individual druse regression timepoint is defined in an automated manner as the point when the volume drops and stays at below $10 \%$ of its baseline value. In our study population, $11 \%$ of individual drusen regressed within the 12 months. Only drusen larger than $0.001 \mathrm{~mm}^{3}$ and within $5 \mathrm{~mm}$ circle around fovea are considered, as done similarly in $[2,10]$. The prediction performance was evaluated using leave-one-patient-out cross-validation. The results are reported as the area under the receiver operating characteristic (ROC) curve (AUC). The feature importance is measured during the random forest training as the increase in prediction error if the values of that feature are permuted across the out-of-bag observations.

The ten most important features for prediction are shown in Fig. 6(a). It can be observed that HRF-based features played an important role and appear in six of the top ten features. This is consistent with the reports from natural history observations [3]. We have built and evaluated different predictive models using a subset of features to further measure the impact of intensity-based and HRF-based features on the performance (Fig. 6(b)). The performance increased monotonically with the addition of the intensity and HRF features obtaining the AUC of 0.73 (shape only), 0.76 (shape + intensity), and 0.81 (shape + intensity + HRF). Setting the operating point that maximizes both the sensitivity and specificity produced a sensitivity of 0.74 and a specificity of 0.73 .

\section{Summary and Conclusion}

Currently there is no treatment available for nonexudative AMD and the disease progresses slowly. Moreover individual progression rates are markedly heterogeneous. Thus, knowing which subjects are at a higher risk of disease regression and hence of late AMD development would find its application in clinical practice as well as designing the clinical trials. In this paper we built and evaluated a predictive model at the level of an individual drusen and showed that predicting drusen regression is feasible. To the best of our knowledge this is the first time a model predicting regression of a druse has been proposed. In addition, 


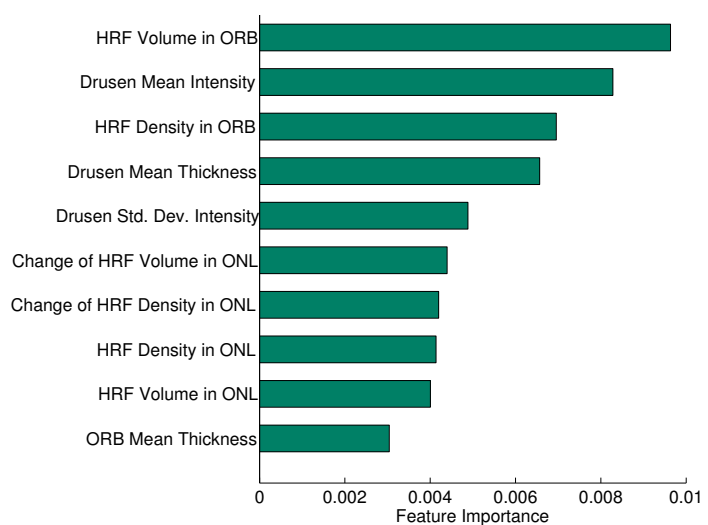

(a)

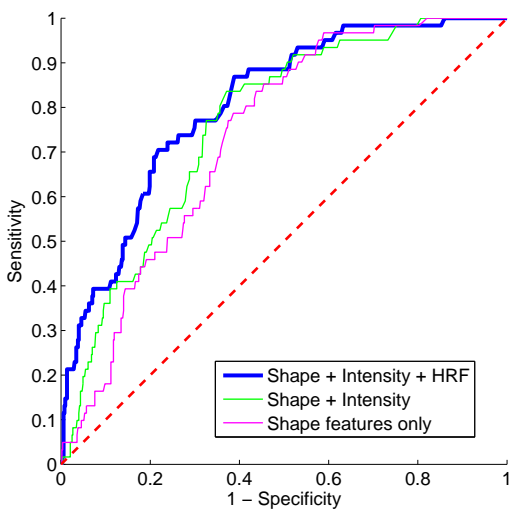

(b)

Fig. 6: (a) Top ten most important features. (b) ROC curves of predictive models built with different feature subsets.

we confirmed that HRF does have an important predictive role. We benefited from a clinical dataset which contained 3-month follow-ups, which is unusually frequent, allowing to compute differential features and identify the regression event with greater precision.

This study has several limitations, most notably the small number of patients in the dataset. This is partly due to the difficulty in identifying and recruiting patients for a clinical study because early and intermediate stages of AMD do not yet hinder patients' vision. In addition, we identified drusen footprints at baseline and kept them fixed, hence not accounting for possible drusen area expansion with time. Finally, the regression event serving as target for learning and testing the prediction has been identified in an automated manner, which is susceptible to segmentation inaccuracies.

In general as drusen grow they merge with neighboring ones to create a confluent drusen. Regression of one part of such drusen causes the entire confluent drusen to regress. Here we model each part of confluent drusen independently. The exploitation of structural information and interaction with neighboring drusen is a subject of future work.

This preliminary study is a promising step toward identification of imaging biomarkers of incoming regression which develops within a predictable and clinically relevant time frame, thus facilitating the development and evaluation of novel interventions that target early and intermediate stages of AMD.

Acknowledgments. The financial support of the Austrian Federal Ministry of Economy, Family and Youth, and the National Foundation for Research, Technology and Development is gratefully acknowledged. 


\section{References}

1. Schaal, K.B., Rosenfeld, P.J., Gregori, G., Yehoshua, Z., Feuer, W.J.: Anatomic clinical trial endpoints for nonexudative age-related macular degeneration. Ophthalmology 123(5) (May 2016) 1060-79

2. Yehoshua, Z., Wang, F., Rosenfeld, P.J., Penha, F.M., Feuer, W.J., Gregori, G.: Natural history of drusen morphology in age-related macular degeneration using spectral domain optical coherence tomography. Ophthalmology 118(12) (December 2011) 2434-41

3. Ouyang, Y., Heussen, F.M., Hariri, A., Keane, P.A., Sadda, S.R.: Optical coherence tomography-based observation of the natural history of drusenoid lesion in eyes with dry age-related macular degeneration. Ophthalmology 120(12) (December 2013) 2656-65

4. Wu, Z., Luu, C.D., Ayton, L.N., Goh, J.K., Lucci, L.M., Hubbard, W.C., Hageman, J.L., Hageman, G.S., Guymer, R.H.: Optical coherence tomography-defined changes preceding the development of drusen-associated atrophy in age-related macular degeneration. Ophthalmology 121(12) (December 2014) 2415-22

5. Gregori, G., Wang, F., Rosenfeld, P.J., Yehoshua, Z., Gregori, N.Z., Lujan, B.J., Puliafito, C.A., Feuer, W.J.: Spectral domain optical coherence tomography imaging of drusen in nonexudative age-related macular degeneration. Ophthalmology 118(7) (July 2011) 1373-9

6. Chiu, S.J., Toth, C.A., Bowes Rickman, C., Izatt, J.A., Farsiu, S.: Automatic segmentation of closed-contour features in ophthalmic images using graph theory and dynamic programming. Biomed. Opt. Express 3(5) (2012) 1127-40

7. Chen, Q., Leng, T., Zheng, L., Kutzscher, L., Ma, J., de Sisternes, L., Rubin, D.L.: Automated drusen segmentation and quantification in SD-OCT images. Med. Image Anal. 17(8) (December 2013) 1058-72

8. Dufour, P.A., Ceklic, L., Abdillahi, H., Schroder, S., De Dzanet, S., WolfSchnurrbusch, U., Kowal, J.: Graph-based multi-surface segmentation of OCT data using trained hard and soft constraints. IEEE Trans. Med. Imag. 32(3) (2013) 531-543

9. de Sisternes, L., Simon, N., Tibshirani, R., Leng, T., Rubin, D.L.: Quantitative SD-OCT imaging biomarkers as indicators of age-related macular degeneration progression. Invest. Ophthalmol. Vis. Sci. 55(11) (2014) 7093-7103

10. Folgar, F.A., Yuan, E.L., Sevilla, M.B., Chiu, S.J., Farsiu, S., Chew, E.Y., Toth, C.A.: Drusen volume and retinal pigment epithelium abnormal thinning volume predict 2-year progression of age-related macular degeneration. Ophthalmology 123(1) (January 2016) 39-50.e1

11. Lee, K., Niemeijer, M., Garvin, M.K., Kwon, Y.H., Sonka, M., Abramoff, M.D.: Segmentation of the optic disc in 3-D OCT scans of the optic nerve head. IEEE Trans. Med. Imag. 29(1) (2010) 159-168

12. Chen, X., Niemeijer, M., Zhang, L., Lee, K., Abramoff, M.D., Sonka, M.: Threedimensional segmentation of fluid-associated abnormalities in retinal OCT: probability constrained graph-search-graph-cut. IEEE Trans. Med. Imag. 31(8) (2012) 1521-31

13. Montuoro, A., Simader, C., Langs, G., Schmidt-Erfurth, U.: Rotation invariant eigenvessels and auto-context for retinal vessel detection. In Ourselin, S., Styner, M.A., eds.: SPIE Medical Imaging. (March 2015) 94131F

14. Tu, Z., Bai, X.: Auto-context and its application to high-level vision tasks and 3D brain image segmentation. IEEE Trans. Pattern Anal. Mach. Intell. 32(10) (October 2010) 1744-1757 